\title{
Analysis of Progressively Unbalanced Induction Motor Current Signals Based on Information Entropy
}

\author{
Ignas Andrijauskas, Rimas Adaskevicius \\ Department of Electric Power Systems, Kaunas University of Technology, \\ Studentu St. 48-144a, LT-51367 Kaunas, Lithuania \\ ignas.andrijauskas@ktu.edu
}

\begin{abstract}
The article presents the capabilities of a new fault identification method used for different fault levels. The method allows identifying unbalanced induction motor by only using stator current signals. The signal analysis was done by using wavelet packet decomposition and reconstruction (WPDR) and information entropy methods. The validation of proposed method was carried out by comparing unbalanced fault progressive simulation and experimentally obtained results. The experimental results were also analysed to identify the most band of frequencies (node) for the proposed method. Signals were divided into five overlapped time intervals in order to investigate which interval is the most informative for fault diagnosis.
\end{abstract}

Index Terms-Induction motors; Information entropy; Unbalance; Wavelet packets.

\section{INTRODUCTION}

Nowadays the most popular type of motor in industry use is the squirrel-cage induction motor (IM). These type motors consume $40-50 \%$ of generated electrical power in the World [1]-[3]. In recent years, the field of IM has been playing a very important part in the development of certain emerging technologies such as renewable energy or electric vehicles.

As any type of devices, the motors are also subject to various faults. For this reason, unscheduled inactivity of IM is a significant problem of the industry worldwide. The reasons for that are costly machinery repairs, extended processes downtime, health and safety problems. Around $50 \%$ of the faults in rotating machines are bearing related, $10 \%$ are rotor faults. The unbalance is within the $12 \%$ of other faults [4], which demonstrates that it is not the most common failure.

In [5] authors get successful results, the certainty of more than $99.7 \%$, by diagnosing three different faults using wavelet packet transform and information entropy analysis of start-up transient phase current signal. Multi-Scale Permutation Entropy-based approach is an effective way to extract the statistical data from the phase current signal of the motor [6]. In this work five different faults: single phasing, single line to ground fault, over-voltage, undervoltage and voltage unbalancing are discussed. All five faults have an electrical origin, but analysis of electrical current signal for diagnosis of mechanical fault is not

Manuscript received 28 September, 2017; accepted 25 April, 2018. presented. Some authors analyse additional signals (currents, axial and radial vibrations) to improve the reliability of fault diagnosis [7]. In [8] the authors propose an optical method to diagnose shaft misalignment, which has an impact on bearings along with surface deformities. A detailed analysis of vibration signals is presented in [9]. Authors investigate dynamic eccentricity in IM by analysing frequency spectrum of signals provided by five three-axes vibration sensors. Although a significant increase of vibration component in case of rotor eccentricity is clearly visible, further investigation, with additional measurements of acoustic vibrations is needed. In order to detect rotor eccentricity authors in [10] propose to use additional Hall effect sensor mounted inside IM. Despite installation inconvenience, this method showed immunity for non-ideal load applications.

It is well-known fact that more complex system is more accurate. But reliability is decreasing with every additional element in a system.

Another fault detection approach is to use stator current signal. Different fault produces different frequency component in signal spectrum. In the study [11] is presented that three faults produce relevant components. The emergence of frequency components is explained in detail. Fast Fourier transform (FFT) is a common implementation for motor current signature analysis. However, some authors [12] claim to face a problem using FFT in load varying applications. They propose to use Discrete Wavelet Transform (DWT) to eliminate varying load influence. The advantage of this study is that eccentricity is analysed in a progressive way. Should be noted that apparent power is used for calculations, so measurements of phase currents and voltages are necessary. In [13] authors propose a statistical-based approach for fault detection in IM. The presented technique is based on maximum likelihood estimation approach. Simulation and experimental results have corroborated the efficiency of the proposed method, regardless of the fault type in contrary to the FFT-based approach. It should be noted that for diagnosis rotor speed must be measured. This is often complicated in particularly active technological lines or in hazardous environments. Significant results are given in [14], where authors indicate that the stationary air-gap eccentricity induces additional rotor slot harmonic components. Analytical and simulation 
results were confirmed by different experimental tests. The artificial neural networks (ANN) are also used in IM diagnosis. In [15] accuracy over $93 \%$ is reached by diagnosing short circuit in stator windings by use of stator current signals. The faults were obtained using short circuit levels of $1 \%, 3 \%, 5 \%$, and $10 \%$ of the stator windings. These faults consist in a controlled emulation of an insulation breakage; thus, it is possible to artificially create short circuit faults between turns of the same coil, between coils of the same phase, and also between coils of different phases. 150 features were used as an input vector for a neural network which consists of two layers and 10,20 and 50 neurons in the hidden layer ( 3 trials). The best ANN topology is the multilayer perceptron with 10 neurons in the hidden layer, which obtained classification accuracies over $99 \%$ for the experimental tests.

According to [16] information entropy-based diagnostic methods can be used in variable frequency drive (VFD) control scheme, which is very common control scheme in industry. The authors in [17] use wavelet packets to monitor the condition of IM. The advantage of this method is that motor speed measurement is not required.

The motivation of particular study has grown due to three main reasons. Even for [5] method having a great performance, the usage of finite values of entropy for fault identification leads to a narrowed field of approach. There are no results about method reaction to the progression of fault (in this case unbalance). Also, no investigation of the most informative portion of startup time is presented.

The aim of this article is to present and investigate new fault identification method under different conditions, by increasing the level of unbalance in the induction motor. The approach of progressive unbalances allows us to determine which time interval and the band of frequencies are best for diagnosis.

\section{Proposed Method}

\section{A. Mathematical Background}

Wavelet Transform (WT) is a highly accurate mathematical tool for digital signal analysis. WT represents a signal through a linear combination of their basis function. The wavelets must be oscillating, limited in duration and their mean must be zero. The discrete wavelet packet transform was introduced by Coifman, Meyer and Wickerhauser [18] by generalizing the link between multiresolution approximations and wavelets. In other words, it is a powerful tool to divide the signal into frequency subbands. For $j$ levels of decomposition, the wavelet packet decomposition produces $2^{j}$ different sets of coefficients. The diagram of wavelet packet transform at level 2 is presented in Fig. 1, where $i_{\mathrm{a}}(t)$ is analysed signal, $i_{\mathrm{a}(j, k)}$ is coefficients, $j$ and $k$ are integer numbers that represent the number of decomposition level and translation, respectively, $h(k)$ and $g(k)$ are wavelet quadrature mirror filters (QMF).

QMF depends on mother wavelet. In this paper Daubechies 5 (db5) wavelet function is used as mother wavelet. Corresponding filter coefficients are presented in Fig. 2.

At the first stage only scaling and mother wavelet equations are applied

$$
W_{j, k}^{n}(t)=2^{j / 2} W^{n}\left(2^{j} t-k\right),
$$

where $W$ is mother wavelet function, $n$ represents the modulation index (the channel number). Then, when $(n=2$, $3, \ldots)$, the wavelet functions can be obtained by:

$$
\begin{gathered}
W_{1, k}^{2 n}(t)=\sqrt{2} \sum_{k} h(k) W_{1, k}^{n}(2 t-k), \\
W_{1, k}^{2 n+1}(t)=\sqrt{2} \sum_{k} g(k) W_{1, k}^{n}(2 t-k), \\
i_{\mathrm{a}(j, k)}=\int i_{\mathrm{a}}(t) W_{j, k}^{n}(t) d(t) .
\end{gathered}
$$

Individual coefficient can be obtained by (4), where $i_{\mathrm{a}}(t)$ in our case is stator current signal of phase A.

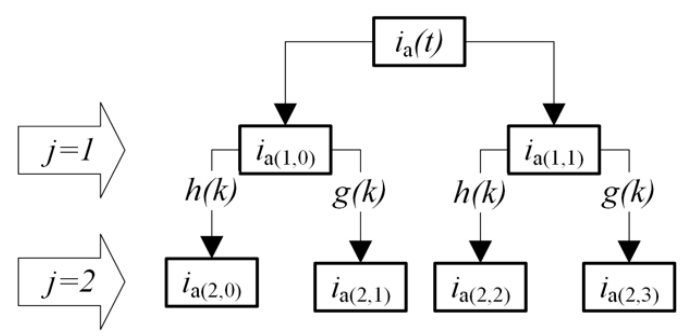

Fig. 1. Diagram of wavelet packet transform.

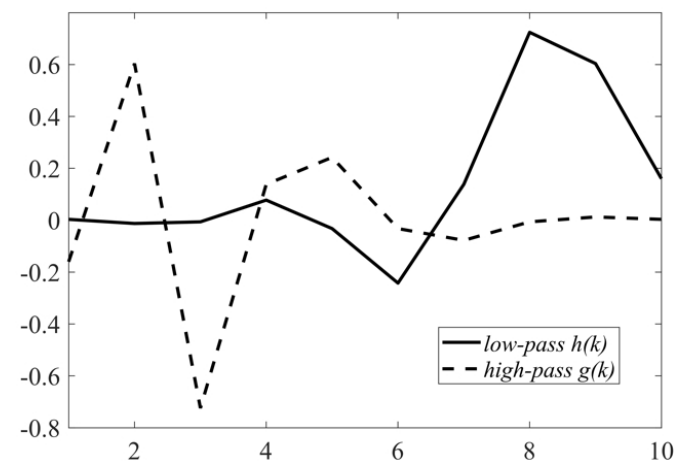

Fig. 2. Daubechies 5 wavelet low-pass and high-pass filters coefficients for decomposition.

In this paper level 2 wavelet packet decomposition and reconstruction (WPDR) were produced. Coefficients (Nodes) are denoted as $i_{\mathrm{a}(j, k)}$ in (4). Nodes and corresponding frequency sub-bands at sampling frequency $f_{\mathrm{s}}=1500 \mathrm{~Hz}$ are presented in Table I.

TABLE I. NODES AND FREQUENCIE SUB-BANDS AT 1500 HZ

\begin{tabular}{|c|c|c|c|c|}
\hline Node & $\boldsymbol{i}_{\boldsymbol{a}(2,0)}$ & $\boldsymbol{i}_{\boldsymbol{a}(2,1)}$ & $\boldsymbol{i}_{\boldsymbol{a}(2,2)}$ & $\boldsymbol{i}_{\boldsymbol{a}(2,3)}$ \\
\hline Frequency $[\mathrm{Hz}]$ & $0-188$ & $188-375$ & $375-563$ & $563-750$ \\
\hline
\end{tabular}

Entropy is used to measure the amount of information which is provided by a system or signal. Boltzmann was the first who began to analyse the level of disorder in a thermodynamic system. Shannon [19] improved Boltzmann's principle and established the information entropy model in information theory. Considering a random event $i_{\mathrm{a}}$ with possible outcomes $i_{\mathrm{a} 1} ; i_{\mathrm{a} 2} ; i_{\mathrm{a} 3} ; \ldots ; i_{\mathrm{a} n}$ and each $i_{\mathrm{a} i}$ with a probability $p\left(i_{\mathrm{a} i}\right)$, then, the information entropy of a random event $i_{\mathrm{a}}$ is given by (5)

$$
E\left(i_{\mathrm{a}}\right)=-\sum_{i=1}^{n} p\left(i_{\mathrm{a} i}\right) \log _{2}\left[p\left(i_{\mathrm{a} i}\right)\right] .
$$


In our case, as random event both a signal of motor inrush current $\left(i_{\mathrm{a}}\right)$ and specific frequency sub-band signal $\left(i_{\mathrm{a}(j, k)}\right)$ were used. An entropy $E$ of this signal is used to evaluate different levels of unbalance. The combination of WPDR and information entropy is chosen because of capability to split signal into frequency sub-bands and measure the amount of information in the signal. The algorithm is discussed in next subsection.

\section{B. Algorithm}

The following steps of the algorithm are used to diagnose the fault:

1. Acquisition of stator phase current $\left(i_{\mathrm{a}}\right)$ signal;

2. Calculation of information entropy of signal $\left(E\left(i_{\mathrm{a}}\right)\right)$;

3. Signal division into frequency sub-bands $\left(i_{\mathrm{a}(j, k)}\right)$, using WPDR (2-level);

4. Calculation of information entropy of specific frequency sub-band signal (e.g. $\left.E\left(i_{\mathrm{a}(2,1)}\right)\right)$;

5 . Calculation of ratio between entropies before WPDR $\left(E\left(i_{\mathrm{a}}\right)\right)$ and after WPDR $\left(E\left(i_{\mathrm{a}(2,1)}\right)\right)$;

6. Compare ratio and fault representing threshold values.

The proposed algorithm is presented in Fig. 3.

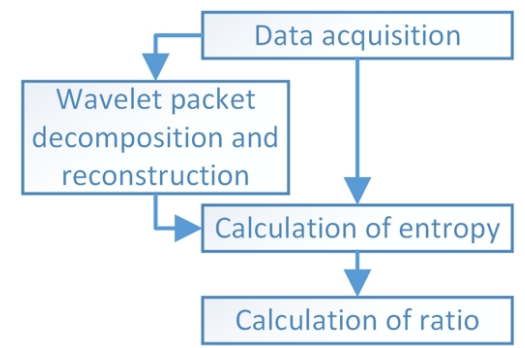

Fig. 3. Flowchart of proposed algorithm.

\section{Experiment}

To validate proposed methodology and define best terms, IM was tested in the laboratory at different levels of unbalance. Motor nameplate data is presented in Table II.

TABLE II INDUCTION MOTOR NAMEPLATE DATA.

\begin{tabular}{|c|c|}
\hline Parameter & Value \\
\hline Power [kW] & 1.1 \\
\hline Voltage [V] & $230 / 400$ \\
\hline Pole pairs & 3 \\
\hline Frequency [Hz] & 50 \\
\hline Rotation speed [rpm] & 1000 \\
\hline
\end{tabular}

During the experiment, one of three stator phase current was sampled. Analogue to digital converter (sampling frequency $-f_{\mathrm{s}}=1500 \mathrm{~Hz}$ ) and current transformer ("YHDC SCT-013-000") were used to capture the phase current signal.

Phase current signals were imported into a PC memory. The data processing and analysis were done by means of our software made in "Matlab". The scheme of the experiment is presented in Fig. 4.

It should be noted that no additional starting equipment was used, the motor was connected directly to an electrical power grid. The experiment comprised two main parts. The first was data acquisition of healthy and progressively unbalanced IM, whereas the second one was procession of the data for explicit results. To imitate IM unbalance, additional weights (see Table III) were fastened on the pulley (Fig. 5). Total of 11 cases are investigated (balanced
IM and 10 cases of unbalances).

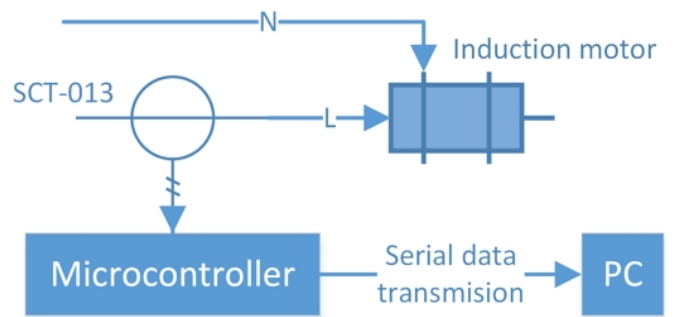

Fig. 4. Scheme of the experiment.

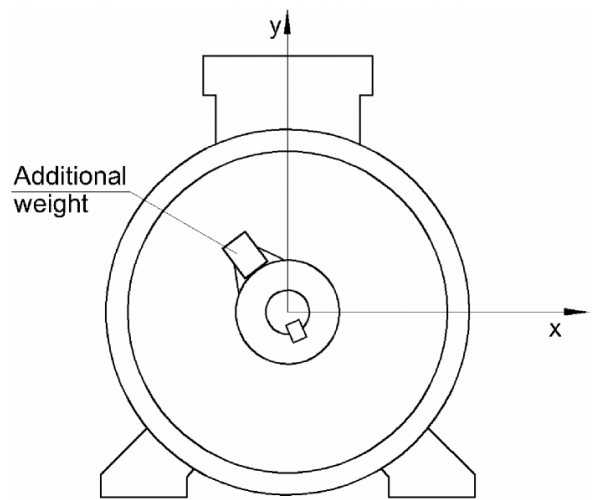

Fig. 5. The front view of IM with attached additional weight on pulley.

Data acquisition was started with balanced IM (no additional weights). The case of balanced IM was used as a reference signal.

\begin{tabular}{|c|c|c|}
\hline TABLE III. ADDITIONAL WEIGTHS USED TO UNBALANCE IM. \\
\hline No. of case & Weight [g] & Total additional weight [g] \\
\hline 1 & 5 & 5 \\
\hline 2 & 7 & 12 \\
\hline 3 & 6 & 18 \\
\hline 4 & 6 & 24 \\
\hline 5 & 7 & 31 \\
\hline 6 & 6 & 37 \\
\hline 7 & 7 & 44 \\
\hline 8 & 5 & 49 \\
\hline 9 & 6 & 55 \\
\hline 10 & 7 & 62 \\
\hline
\end{tabular}

The second and other subsequent acquisitions of data were produced using additional weights. To reduce uncertainties, the signal of each case was captured and investigated 10 times. A total data set of 110 independent signals was collected during the first part of the experiment. For processing, the signals were divided into five overlapping time intervals (see Table IV). Duration of each analysed signal was $3 \mathrm{~s}$. In order to extract signal from IM start the software was developed. The signals were extracted from the time when the inrush current amplitude reaches the determined threshold.

TABLE IV. TIME INTERVALS.

\begin{tabular}{|c|c|}
\hline \multicolumn{1}{|c|}{ TABLE IV. TIME INTERVALS. } \\
\hline No. & Time intervals [s] \\
\hline I & $0-1$ \\
\hline II & $0.5-1.5$ \\
\hline III & $1-2$ \\
\hline IV & $1.5-2.5$ \\
\hline V & $2-3$ \\
\hline
\end{tabular}

One of the analysed signals with two time intervals (I and II) is displayed in Fig. 6. Because of overlapping time intervals, it can be determined which interval is the best for the diagnosis of a broken motor. 


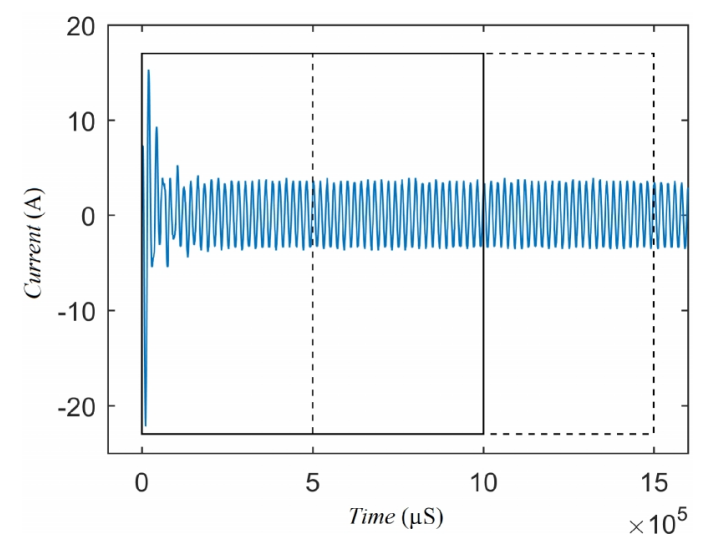

Fig. 6. One of current signals, with marked time intervals I (solid line) and II (dashed line).

When signals are prepared, WPDR of level 2 can be performed. The next step is a calculation of Shannon's entropy. The entropy of signal before WPDR is selected as a reference. The ratio between base signal entropy and entropy of signal after WPDR was calculated. The progressively held experiment showed system reactions. For convenience, results are presented in box plots (Fig. 7, Fig. 8) where circle with dot represents median value of entropies ratio.

\section{RESULTS}

Figure 7 shows, that median entropy ratio of a investigated healthy motor is around 1.785 . Only in a few cases (when $37 \mathrm{~g}$ and $55 \mathrm{~g}$ weights were applied, Table III) there is a clear difference, without overlapping, in values of lower and upper quartile between healthy and broken motor, while in the remaining cases impossible to make the correct decision.

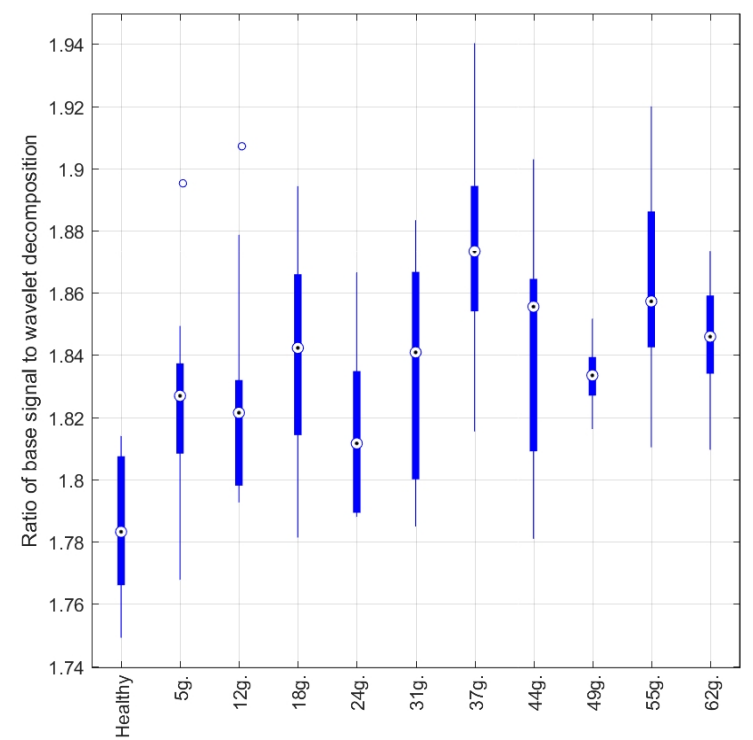

Fig. 7. Ratio of a base signal to wavelet decomposition node 2,1 at time interval II.

In Fig. 7 and Fig. 8 balanced IM case is labelled as healthy.

Figure 8 shows, that median entropies ratio of a healthy motor is around 1.73. In all cases, there is a clear difference in the value of a lower and upper quartile between healthy and broken motors.

For example, the worst case is when the $12 \mathrm{~g}$ weight was used (case 2) because it has the lowest contrast. Conversely, usage of $37 \mathrm{~g}$ weight (case 6) has the highest contrast. It is worth noting that the smallest $5 \mathrm{~g}$ weight (case 1) causes the fault which has a very high contrast, like using the $37 \mathrm{~g}$ weight (case 6).

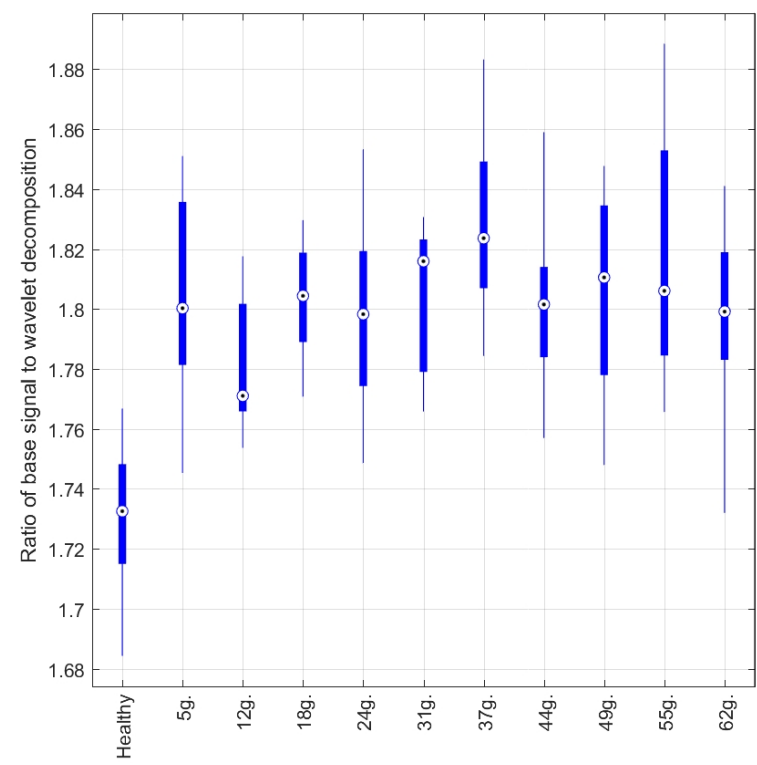

Fig. 8. Ratio of base signal to wavelet decomposition node 2,2 at time interval $\mathrm{lV}$.

\section{A. Discussion}

The analysis of results shows that the best node for diagnosis is 2,2. It should be noted that node 2,2 has a high contrast between healthy and broken motors (except time interval IV, when one of the cases is undiagnosable). It can be noted that in all nodes interval I has demonstrated the worst results. Interval with the best performance at node 2,2 is $\mathrm{V}$ (Fig. 8) when the difference between values of a lower and upper quartile of healthy and broken motors respectively is around 0.02 . It is worthwhile noting, that during the experiment, unbalance of $37 \mathrm{~g}$ (case 6) and $44 \mathrm{~g}$ (case 7) was felt more than in other cases. This is also reflected in the results in case 6 (Fig. 8).

It is evident that using the right node and time interval, the proposed methodology is sensitive enough for discovering even relatively small unbalances in IM.

\section{CONCLUSIONS}

This paper presents a comparison of balanced and progressively unbalanced induction motor inrush current signals. The method is based on a calculation of the ratio between information entropy before and after wavelet packet decomposition and reconstruction of a current signal. The progressively held experiment has proved that the proposed methodology is suitable both for low and high levels of unbalance. During the experiment, best frequency band and time intervals for diagnosis were determined $-f=$ [375-563] $\mathrm{Hz}$ and $t=[1.5-3] \mathrm{s}$. The results have also demonstrated that information entropy ratio is a nonlinear function of unbalance level. Time intervals of the sampled signal and the nodes of decomposition ware analysed and discussed. Sensitive mechanical fault diagnosis based on electrical signal processing is a key advantage of the proposed method. 


\section{REFERENCES}

[1] M. E. H. Benbouzid, "A review of induction motors signature analysis as a medium for faults detection", IEEE Trans. Industrial Electronics, vol. 47, pp. 984-993, 2000. DOI: 10.1109/41.873206.

[2] S. A. S. Al-Kazzaz, G. K. Singh, "Experimental investigations on induction machine condition monitoring and fault diagnosis using digital signal processing techniques", Electric Power Systems Research, vol. 65, pp. 197-221, 2003. DOI: 10.1016/S03787796(02)00227-4.

[3] W. T. Thomson, M. Fenger, "Current signature analysis to detect induction motor faults", IEEE Industry Application Magazine, vol. 26-34. DOI: 10.1109/2943.930988.

[4] R. J. Romero-Troncoso, R. Saucedo-Gallaga, E. Cabal-Yepez, "FPGA-based online detection of multiple combined faults in induction motors through information entropy and fuzzy inference", IEEE Trans. Industrial Electronics, vol. 58, no. 11, 2011. DOI: 10.1109/TIE.2011.2123858.

[5] E. Cabal-Yepez, R. J. Romero-Troncoso, A. Garcia-Perez, R. A. Osornio-Rios, "Single-parameter fault identification through information entropy analysis at the startup-transient current in induction motors", Electric Power Systems Research, vol. 89, pp. 6469, 2012. DOI: 10.1016/j.epsr.2012.02.016.

[6] P. S. Bhowmik, M. Prakash, S. Pradhane, "A novel neuro-classifier using multiscale permutation entropy for motor fault diagnosis", in IEEE Conf. Control Applications (CCA 2014), 2014. DOI: 10.1109/CCA.2014.6981374.

[7] A. Han li, Shi Li-ping, "Fault diagnosis of induction motor based on information entropy fusion", in 2nd Int. Conf. Advanced Computer Control (ICACC 2010), 2010. DOI: 10.1109/ICACC.2010.5486779.

[8] A. G.Fulzele, V. G. Arajpurea, P. P. Holayb, N. M. Patil, "Condition monitoring of shaft of single-phase induction motor using optical sensor", Mechanical Systems and Signal Processing, vol. 29, pp. 428435, 2012. DOI: 10.1016/j.ymssp.2011.12.002.

[9] J. Sobra, T. Vaimann, A. Belahcen, "Mechanical vibration analysis of induction machine under dynamic rotor eccentricity", in IEEE 17th Int. Scientific Conf. Electric Power Engineering (EPE 2016), 2016. DOI: 10.1109/EPE.2016.7521732.

[10] Y. Park, D. Fernandez, S. B. Lee, D. Hyun, M. Jeong, S. K. Kommuri, C. Cho, D. Reigosa, F. Briz, "On-line detection of rotor eccentricity for PMSMs based on hall-effect field sensor measurements", in IEEE Energy Conversion Congress and Exposition (ECCE 2017), 2017. DOI: 10.1109/ECCE.2017.8096798.

[11] I. Ishkova, O. Vitek, "Diagnosis of eccentricity and broken rotor bar related faults of induction motor by means of motor current signature analysis", in IEEE1 6th Int. Scientific Conf. Electric Power Engineering (EPE 2015), 2015. DOI: 10.1109/EPE.2015.7161130.

[12] K. Yahiaab, A. J. M. Cardosob, A. Ghoggalc, S. E. Zouzou, "Induction motors airgap-eccentricity detection through the discrete wavelet transform of the apparent power signal under non-stationary operating conditions", ISA Trans., vol. 53, no. 2, pp. 603-611, 2014. DOI: $10.1016 /$ j.isatra.2013.12.002.

[13] El Houssin, El Bouchikhiab, V. Choqueusea, M. Benbouzid, "Induction machine faults detection using stator current parametric spectral estimation", Mechanical Systems and Signal Processing, vol. 52-53, pp. 447-464, 2015. DOI: 10.1016/j.ymssp.2014.06.015.

[14] M. El K. Oumaamar, Y. Maouche, M. Boucherma, A. Khezzar, "Static air-gap eccentricity fault diagnosis using rotor slot harmonics in line neutral voltage of three-phase squirrel cage induction motor", Mechanical Systems and Signal Processing, vol. 84, Part A, pp. 584 597, 2017. DOI: 10.1016/j.ymssp.2016.07.016.

[15] G. H. Bazanab, P. R. Scalassaraa, W. Endoa, A. Goedtela, W. F. Godoya, R. H. C. Palacios, "Stator fault analysis of three-phase induction motors using information measures and artificial neural networks", Electric Power Systems Research, vol. 143, pp. 347-356, 2017. DOI: 10.1016/j.epsr.2016.09.031.

[16] E. Cabal-Yepez, R. J. Romero-Troncoso, A. Garcia-Perez, R. A Osornio-Rios, R. Alvarez-Salas, "Multiple fault detection through information entropy analysis in ASD-fed induction motors", in IEEE International Symposium Diagnostics for Electric Machines, Power Electronics \& Drives (SDEMPED 2011), 2011. DOI: 10.1109/DEMPED.2011.6063653.

[17] D. Gokhan Ece, M. Basaran, "Condition monitoring of speed controlled induction motors using wavelet packets and discriminant analysis", Expert Systems with Applications, vol. 38, pp. 8079-8086, 2011. DOI: 10.1016/j.eswa.2010.12.149.

[18] R. R. Coifman, Y. Meyer, M. V. Wickerhauser, "Wavelet analysis and signal processing", Wavelets and their Applications, pp. 153-178, Boston, 1992.

[19] C. E. Shannon, "A mathematical theory of communication", The Bell System Technical Journal, vol. 27, pp. 379-423, 1948. DOI: j.15387305.1948.tb00917.x. 